\title{
ESTUDIO DE IMPACTO ARQUEOLÓGICO EN PUNTA PEREIRA (COLONIA- URUGUAY): METODOLOGÍA APLICADA Y PRINCIPALES RESULTADOS PARA EL CONOCIMIENTO DE LA PREHISTORIA REGIONAL.
}

\begin{abstract}
Archaeological impact assessment at Punta Pereira (Colonia County, Uruguay): methodological aspects and main results for regional prehistory knowledge.
\end{abstract}

\section{Irina Capdepont ${ }^{1}$ Laura del Puerto e Hugo Inda ${ }^{2}$}

\section{RESUMO}

Este trabalho apresenta os resultados obtidos no estudo de avaliação e diagnóstico de impacto arqueológico na zona de Punta Pereira (Departamento de Colonia - Uruguai). Numa primeira etapa, as diferentes áreas da zona de Punta Pereira com potencial arqueológico foram identificadas, localizadas, descritas, documentadas, estudadas e valorizadas. Posteriormente, com o objetivo de mitigar o impacto que seria produzido pelas obras planificadas pela Planta de Celulosa y Energia Elétrica, definiu-se o entorno de proteção de cinco espaços com evidências de atividade humana pré-histórica. Nestes espaços se aplicaram medidas corretoras compensatórias, que incluíram a realização de 12 escavações arqueológicas. Estas etapas de trabalho permitiram registrar ocupações humanas durante o Holoceno que eram desconhecidas até o momento.

Palavras-chave: Impacto Arqueológico, Ocupações humanas pré-históricas.

\begin{abstract}
Results from archaeological impact appraisal and diagnose studies performed at Punta Pereira (Colonia county, Uruguay) were presented in this contribution. Several areas with archaeological potential were identified, spatially referenced, described and studied. In order to mitigate the impact of the Cellulose Processing and Electric Energy Plant construction five areas with prehistoric human evidences were defined for further research. In those areas corrective compensatory measures were applied, including 12 archaeological diggings. Results from such activities allowed to unveil human occupations during middle to late Holocene that were previously unknown to regional Archaeology.
\end{abstract}

Keywords: Archaeological Impact, Prehistorical human occupations.

\section{RESUMEN}

Este trabajo presenta los resultados obtenidos en el estudio de evaluación y diagnóstico de impacto arqueológico de la zona de Punta Pereira (Departamento de Colonia - Uruguay). En una primera instancia se identificaron, localizaron, describieron, documentaron, estudiaron y valoraron diferentes áreas de la zona con potencial arqueológico. Posteriormente, con el objetivo de mitigar el impacto a producirse por las obras planificadas por la Planta de Celulosa y Energía Eléctrica, se definió el entorno de protección de cinco áreas con

\footnotetext{
${ }^{1}$ Laboratorio de Estudios del Cuaternario (MEC-UNCIEP) iracap@yahoo.com.ar

${ }^{2}$ Centro Universitario Regional Este, Universidad de la República
} 
evidencias de actividad humana prehistórica. Sobre estas áreas se aplicaron mediadas correctoras compensatorias que incluyeron la realización de 12 excavaciones arqueológicas. Estas instancias de trabajo permitieron registrar ocupaciones humanas durante el Holoceno no conocidas con anterioridad.

Palabras clave: Impacto Arqueológico, Ocupaciones humanas prehistóricas

\section{Introducción}

El Patrimonio Arqueológico está constituido por todos los restos físicos tangibles de la acción humana del pasado que contienen información sobre ésta. Es debido a su carácter de bien de interés público, frágil y no renovable, que la Ley de Impacto Ambiental ( $\mathrm{N}^{\circ} 16.466$ Decreto 435/994) en Uruguay exige la realización de Estudios de Impacto Arqueológico (EIArq) a fines de diagnosticar, prevenir, corregir y/o mitigar los efectos negativos de distintos emprendimientos públicos o privados sobre los bienes arqueológicos. En este contexto, la proyección e implementación de una planta de fabricación de celulosa $\mathrm{y}$ generación de energía eléctrica en Punta Pereira, sobre el litoral oeste de Uruguay (Figura 1), constituyó el marco para conjugar el estudio de impacto como iniciativa de investigación para la generación de conocimientos. Dado que el proyecto productivo ya contaba con la habilitación ambiental para la localización de la planta, el Estudio de Impacto Arqueológico

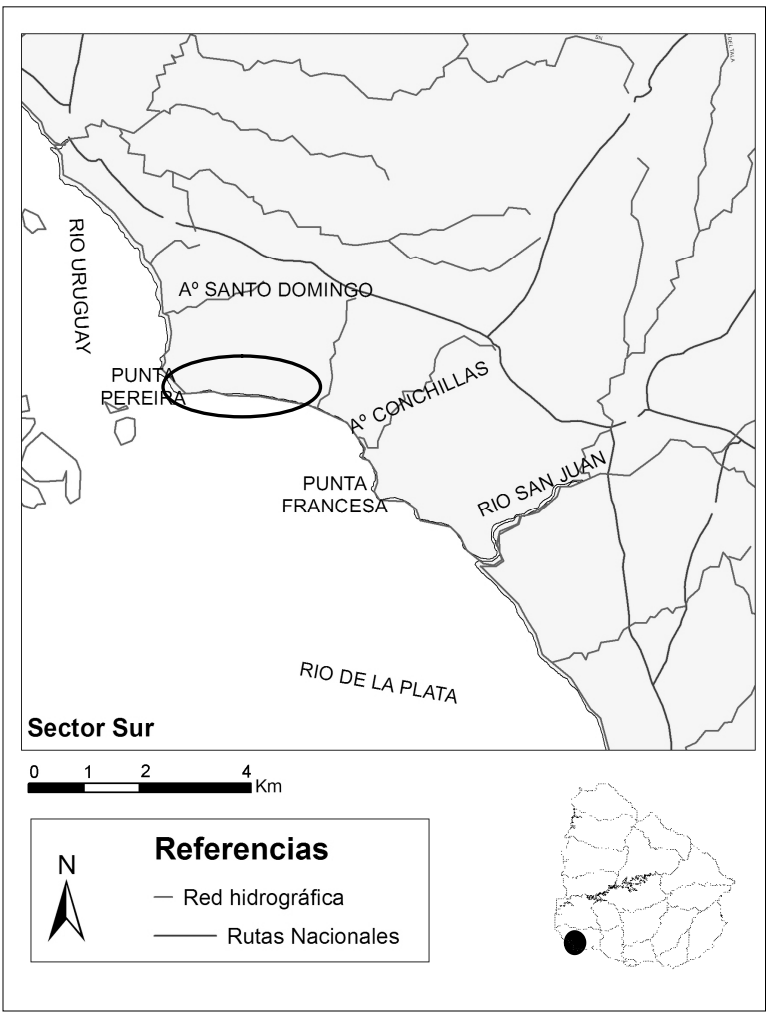
se desarrolló principalmente con miras a diagnosticar y mitigar el impacto de las obras proyectadas. Para ello, en una primera instancia se realizó la Evaluación del Impacto con la finalidad de identificar objetos o elementos patrimoniales y definir sus entornos de protección antes del comienzo de las obras. Esta instancia involucró (sensu AMADO et al., 2002) el descubrimiento, localización, descripción, documentación, estudio, valoración y difusión de los valores culturales allí presentes. Posteriormente, con el objetivo de mitigar el impacto a ser producido por la obra proyectada, se llevaron adelante

Fig.1: Ubicación general y específica del área de estudio (Fuente: CAPDEPONT 2012). 
medidas de corrección compensatorias (documentación, prospección y excavación) y paliativas (seguimiento de obra y actuaciones puntuales de recuperación parcial). En este trabajo se presentan los resultados obtenidos de la prospección arqueológica y de las excavaciones efectuadas en dos de los cinco espacios con evidencias de ocupación humana en Punta Pereira (M8 y M6).

\section{Actividades de evaluación y diagnóstico}

La etapa de Evaluación del Impacto involucró actividades específicas que llevaron a realizar el Diagnóstico Arqueológico del área a ser afectada. En esta etapa se llevó a cabo:

Identificación de Afecciones requirió la identificación precisa de los agentes (infraestructura e instalaciones), acciones (actividades concretas que generen impacto), afecciones (modificaciones del medio físico) y momento del impacto (fase del proyecto donde el impacto se hará efectivo). Para el caso de estudio se identificaron tanto afecciones preexistentes como proyectadas, así como los principales agentes, acciones y momento del impacto (Tabla 1).

Al inicio de la investigación, en el predio destinado al emprendimiento se identificó la existencia de afecciones preexistentes, de diversa magnitud y con disímil impacto constatado sobre las entidades arqueológicas. Entre ellas se destacan, por su extensión, los movimientos de suelo producto de las actividades extractivas realizadas por la empresa inglesa C.H. Walker \& Co. que se instaló en la zona entre 1886 y 1887 . La empresa extrajo y exportó arena y conchilla a la ciudad de Buenos Aires, aumentando la extracción hacia 1895. La explotación de áridos fue continuada por la empresa Roselli Importación S.A, cuyas actividades extractivas a partir de 1957, debido a la maquinaria empleada y la escala espacial de la explotación, constituyeron el principal agente de impacto identificado en el área de Punta Pereira (LEZAMA et al., 2007).

Dentro de las acciones proyectadas por el plan de obra (EsIA EDARIX S.A, DINAMA Exp 2007/14000/05626) en la Tabla 1 se señalan las de mayor potencial de afección sobre las entidades arqueológicas. En todos los casos las acciones proyectadas involucraron movimientos de suelo, con empleo de maquinaria pesada de alto impacto, siendo las etapas de nivelación del terreno y terraplenado las de mayor impacto potencial (Figura 2). 
Tabla 1. Principales afecciones preexistentes y proyectadas. (Fuente: LEZAMA et al. 2007)

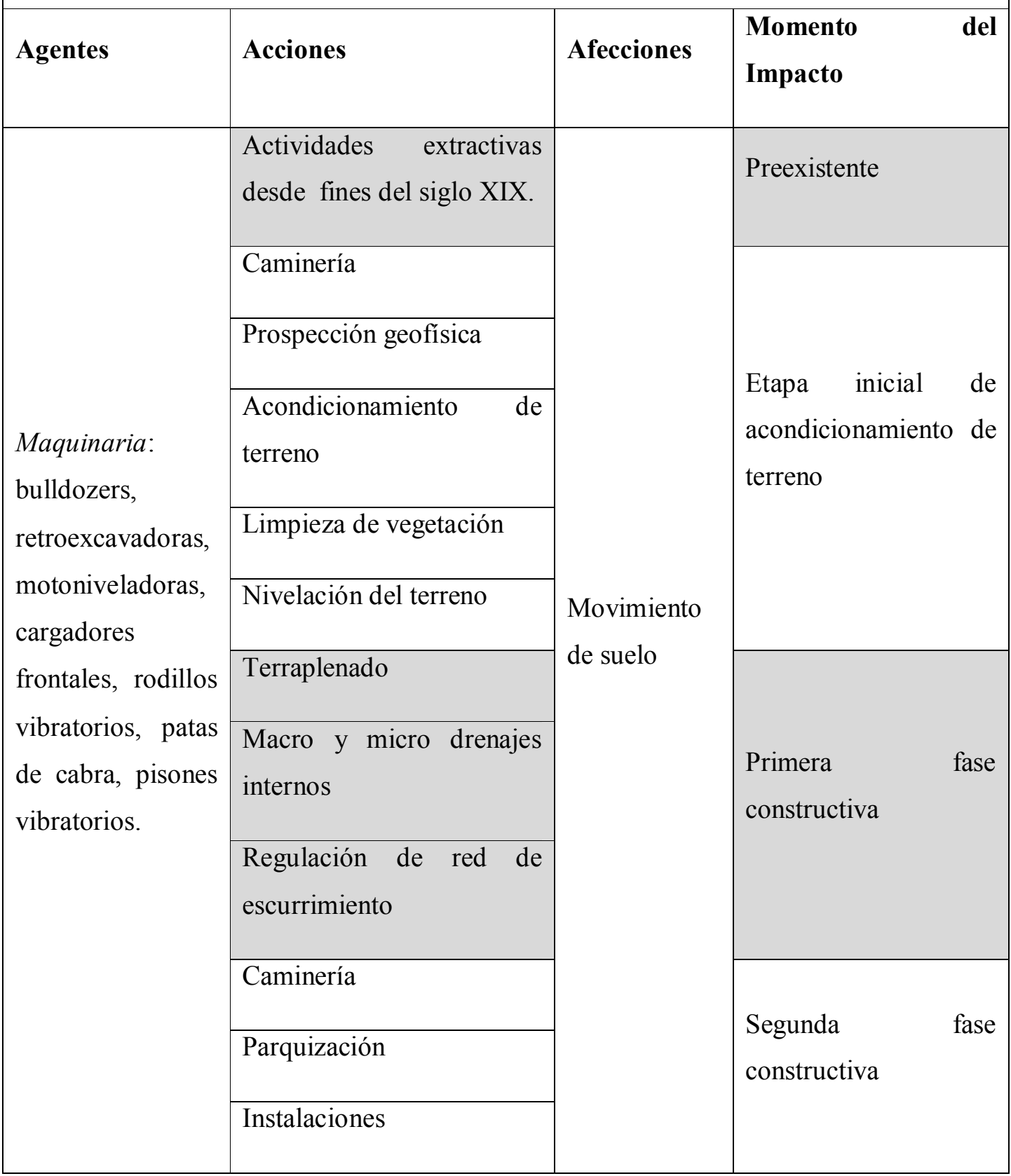




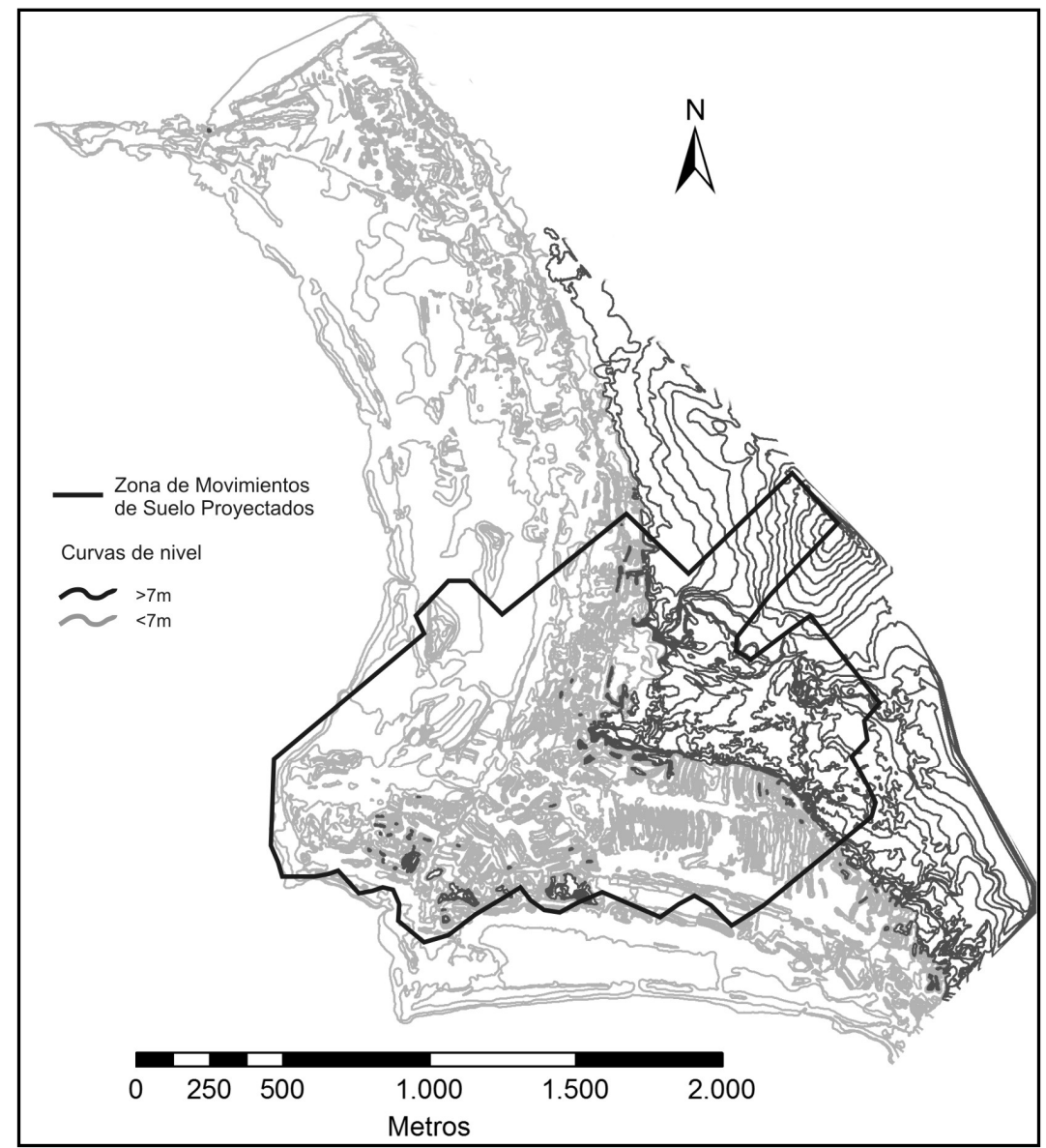

Fig.2: Ubicación de la zona de movimiento de suelo proyectado y referencias topográficas del área de emplazamiento. Los terrenos altos $(>7 \mathrm{~m})$ corresponden a las zonas de desmonte y los terrenos bajos $(<7 \mathrm{~m})$ a las de relleno. Base: Relevamiento topográfico y Plan de Obra Grupo ENCE (Fuente: LEZAMA et al., 2007)

Prospección Arqueológica tuvo como objetivos principales identificar y registrar contextos arqueológicos, efectuando una caracterización primaria que permitiera determinar la existencia de entidades pasibles de ser sujetas a medidas de mitigación. Considerando la heterogeneidad espacial de la información arqueológica, los estudios cartográficos y fotográficos, así como las afecciones producidas, se propuso dividir el área en dos zonas geomorfológicas (CAPDEPONT, 2008): A.- La Zona Baja incluye los sectores Sur, Sudeste y Este del predio, comprendiendo la línea de costa, crestas de tormenta, bañados, aluviones y depósitos arenosos intensamente afectados por la explotación de arena y conchillas. Es de esta porción del terreno de donde deriva la mayoría de los restos arqueológicos que forman hoy día parte del acervo de diversas colecciones tanto públicas como privadas. B.- La Zona Alta comprende el sector noreste del predio, abarcando aquellas fracciones de terreno con cota superior a los $7 \mathrm{msnm}$. El límite sudoeste del área se halla delimitado por la línea de la paleocosta del máximo transgresivo del Holoceno y los depósitos arenosos que la cubren 
parcialmente. En algunos sectores esta paleocosta se ve interrumpida como consecuencia de las actividades extractivas pretéritas (explotación de arena y conchilla desde fines del siglo XIX y principios del siglo XX.), que expusieron en varios puntos contextos arqueológicos relevantes asociados a un paleosuelo areno-limoso interdunar. Dada la riqueza arqueológica registrada en antecedentes y el carácter no alterado de algunos sectores, se identificaron y delimitaron áreas con alto potencial de hallazgo de contextos arqueológicos primarios. El trabajo se centró en el área de ubicación de la Zona Franca (delimitada en la figura 2), donde se proyectaban las mayores obras de movimiento de suelo (CAPDEPONT, 2008).

Para la realización de la etapa de prospección se ejecutó un plan que abarcó dos instancias diferenciales. En primer lugar se llevó a cabo una prospección superficial sistemática pedestre, principalmente en rasgos como escombreras pertenecientes a las antiguas actividades extractivas, caminería, frente expuesto de la escarpa, costa y demás zonas donde la erosión, tanto antrópica como natural, incrementa la visibilidad arqueológica. En forma simultánea, la prospección sistemática se complementó con una prospección dirigida, apuntando a reconocer estructuras específicas. Con ello se atendió a zonas de mayor visibilidad (afloramientos, barrancas, cauces, trillos, etc.) o visibilidad puntual. Las tareas de relevamiento superficial se complementaron con intervenciones arqueológicas puntuales, con el objetivo de indagar el subsuelo en zonas de baja visibilidad arqueológica y detectar la existencia de materiales arqueológicos en estratos sub-superficiales. Estas tareas incluyeron la limpieza de perfiles expuestos, así como la realización de sondeos en una serie de puntos del paisaje, previamente seleccionados de forma selectiva y/o aleatoria. Para ello fue necesario establecer, en primer lugar, puntos de interés y potencial arqueológico, tanto en la zona baja como en la zona alta del predio (CAPDEPONT, 2008; LEZAMA et al., 2008).

Diagnóstico del Impacto, como último paso en la etapa de la Evaluación, es definido de acuerdo a tres criterios: efecto, magnitud e incidencia (AMADO et al., 2002; CRIADO et al., 2000). De ello resultó la definición de los tipos de impacto a generarse en el área: Crítico y Severo. Dado que el desarrollo de la obra genera la desaparición parcial o total de sitios Arqueológicos, se diagnosticó un impacto crítico que implicó la adopción de medidas correctoras destinadas a compensar el impacto a través de un rescate arqueológico. Asimismo, para algunos sectores del área se diagnosticó un impacto severo, permitiendo adoptar medidas preventivas (control y seguimiento de obra) en fase de ejecución de las obras.

Las medidas correctoras planteadas intentaron: recuperar evidencias de las actividades humanas desarrolladas en la zona; obtener un mayor conocimiento de la ocupación humana prehistórica, aportar conocimiento a la arqueología regional y nacional 
mediante la investigación exhaustiva de contextos arqueológicos prehistóricos e históricos a través de metodologías de investigación científica; y finalmente poner en valor los bienes patrimoniales. La primera medida, destinada a la generación de nuevos conocimientos en relación a la ocupación humana de la zona, consistió en la realización de 13 excavaciones de rescate arqueológico en los puntos con alto potencial arqueológico (N8, N9, M8, M6 y 3Pinos) identificados en la primera fase de estudio (CAPDEPONT, 2008 a 2008b; DEL PUERTO, 2008a, 2008b; GASCUE, 2008; LEZAMA et al., 2008).

\section{Resultados del estudio de evaluación}

La presencia de sitios arqueológicos prehistóricos e históricos en diferentes unidades del paisaje, no documentados con anterioridad, ha sido determinada en campo por medio de técnicas de prospección superficial y sub-superficial. Se llevaron a cabo 229 actuaciones en el marco de la prospección arqueológica del área (Tabla 2).

Las actuaciones arqueológicas comprendieron:

a.- Observaciones sin intervención: la mayor parte de estas observaciones da cuenta del registro y relevamiento de distintos movimientos de suelo preexistentes, en los que no se registró presencia de materiales arqueológicos. Se efectuaron 32 actuaciones correspondientes a esta categoría (Tabla 2).

b.- Recolección superficial: el material hallado en superficie fue registrado (fichas de registro de prospección, fotografía, georeferenciación, etiquetado) y acondicionado para su análisis en laboratorio. La mayor parte de estos materiales proviene decontextos secundarios, asociados a movimientos de suelo vinculados a las actividades extractivas pretéritas, erosión natural en los frentes expuestos por la antigua arenera, así como a la construcción y/o acondicionamiento reciente de la caminería. Cabe destacar las recolecciones superficiales efectuadas en la franja costera, que permitieron recuperar materiales cerámicos durante eventos de bajante del nivel del estuario. Fueron recolectados materiales en superficie en 33 locus de la Zona Alta y 32 en la Zona Baja (Tabla 2).

c.- Limpieza de perfiles: se limpiaron y registraron perfiles en frentes expuestos por la antigua extracción de áridos, en depósitos de material de descarte de la arenera, en perforaciones efectuadas por los estudios geológicos previos, en frentes expuestos 
por caminería y obras civiles y en cursos de agua y canales de drenaje. De los perfiles asociados a frentes de explotación de arena, 20 involucran la presencia de un paleosuelo areno-limoso bajo depósitos eólicos de distinta potencia. Al mismo se asocian materiales arqueológicos desplazados en las laderas expuestas y holladas donde se realizaron 43 limpiezas de perfiles (Tabla 2).

d.- Sondeos: se realizaron 79 sondeos (Tabla 2), ubicados en todas aquellas unidades del paisaje en las que resultó factible obtener una buena representación estratigráfica. Como parte de la prospección dirigida, los sondeos realizados en la Zona Alta fueron efectuados junto a la línea de la paleocosta, asociándose en la mayoría de los casos a frentes expuestos por la antigua explotación arenera con un paleosuelo areno-limoso y material cultural. En lo que refiere a la Zona baja, se priorizaron las áreas relictuales no afectadas por la antigua explotación arenera (donde la acumulación de pilas de estéril cubrió depósitos naturales y en parches antiguos de monte) y la costa Sur, aprovechando una bajante extrema del Río de la Plata.

Tabla 2. Detalle y cuantificación por zona de las actuaciones efectuadas en el marco de la prospección arqueológica. (Fuente: modificado de LEZAMA et al. 2008)

\begin{tabular}{|c|c|c|c|c|}
\hline \multicolumn{2}{|l|}{ Actuación } & $\begin{array}{l}\text { Zona } \\
\text { Baja }\end{array}$ & $\begin{array}{l}\text { Zona } \\
\text { Alta }\end{array}$ & $\begin{array}{l}\text { Total de } \\
\text { materiales }\end{array}$ \\
\hline \multirow{3}{*}{ Intervenciones } & Limpieza de Perfil & 13 & 30 & 43 \\
\hline & Sondeo & 14 & 65 & 79 \\
\hline & Rec. Superficial & 32 & 33 & 65 \\
\hline \multirow{5}{*}{ Observaciones directas } & Afloramientos & 0 & 1 & 1 \\
\hline & Caminería & 0 & 3 & 3 \\
\hline & Calicatas & 0 & 12 & 12 \\
\hline & Escombreras & 0 & 4 & 4 \\
\hline & Estructuras & 10 & 1 & 11 \\
\hline
\end{tabular}




\begin{tabular}{|l|l|l|l|l|}
\hline & Forestaciones & 0 & 1 & 1 \\
\hline Total & & 69 & 150 & $\mathbf{2 1 9}$ \\
\hline
\end{tabular}

Las intervenciones mencionadas permitieron recuperar material cultural en contexto primario y secundario (Tabla 3). A partir de la propuesta metodológica desarrollada fue factible definir y delimitar áreas arqueológicas de interés, que serían impactadas de forma crítica y severa por la obra. Ello llevó a delimitar entornos de protección (Figura 3) en los que se realizaron, como medidas correctoras compensatorias, las excavaciones arqueológicas.

\begin{tabular}{|c|c|c|c|c|c|c|}
\hline \multirow{2}{*}{ Intervención } & \multirow{2}{*}{ Contexto } & \multicolumn{4}{|c|}{ Cultura Material Recuperada } & \multirow{2}{*}{ Totales } \\
\hline & & Lítico & Cerámica & Óseo & $\begin{array}{l}\text { Materiales } \\
\text { Históricos }\end{array}$ & \\
\hline \multirow[t]{2}{*}{ Limpieza de Perfil } & Primario & 20 & 0 & 0 & 0 & \multirow[t]{2}{*}{72} \\
\hline & Secundario & 1 & 0 & 0 & 51 & \\
\hline \multirow[t]{2}{*}{ Sondeos } & Primario & 48 & 0 & 1 & 9 & \multirow[t]{2}{*}{62} \\
\hline & Secundario & 4 & 0 & 0 & 0 & \\
\hline $\begin{array}{l}\text { Recolección } \\
\text { Superficial }\end{array}$ & Secundario & 2978 & 60 & 17 & 46 & 3101 \\
\hline \multicolumn{2}{|l|}{ Totales } & 3051 & 60 & 18 & 106 & 3235 \\
\hline
\end{tabular}




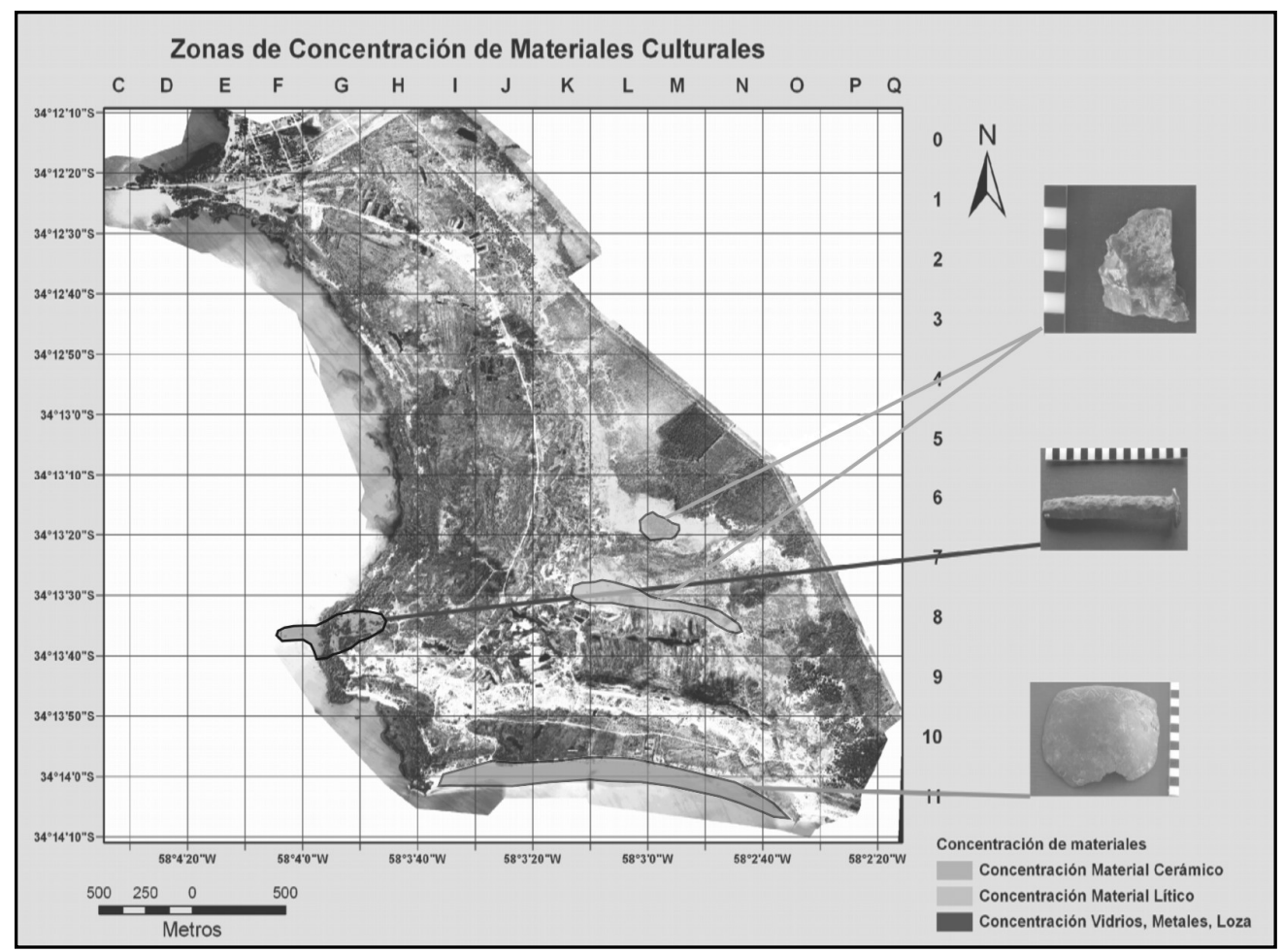

Fig.3: Localización de las principales zonas de concentración de materiales arqueológicos identificadas a partir de la prospección y posteriormente excavadas. Base: Foto Aérea 1:20.000 1981, FAU. (Fuente: LEZAMA et al. 2008).

A partir de las excavaciones realizadas se logró la generación de nuevo conocimiento sobre ocupaciones humanas del Holoceno y se recuperaron evidencias de actividades humanas pretéritas en contexto estratigráfico. Ello aportó a un mayor conocimiento de la arqueología regional y nacional.

Las zonas excavadas corresponden a las áreas denominadas 3 Pinos, M6, M8, N8 y N9 (Figura 4), cuya ubicación geográfica se explicita en la tabla 4. Doce excavaciones se localizaron en la escarpa (límite zona alta y baja; cotas 7-12 msnm) vinculada a la línea de costa durante el máximo transgresivo del Holoceno (N8, N9, M8 y 3 Pinos). La excavación realizada en M6 se ubicó aproximadamente 500 metros al norte de la escarpa, en un espacio interdunar en cota $15 \mathrm{msnm}$. 


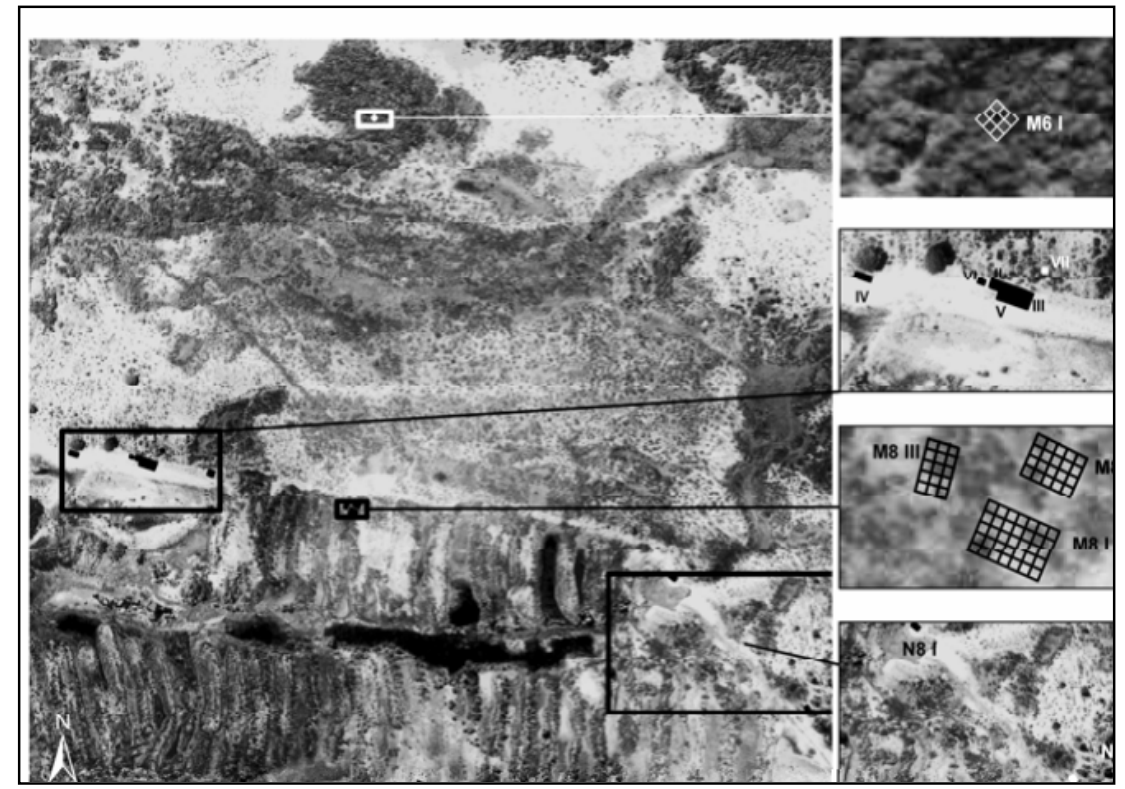

Fig.4:. Fotoplano con áreas excavadas. Fuente: CAPDEPONT 2008:48)

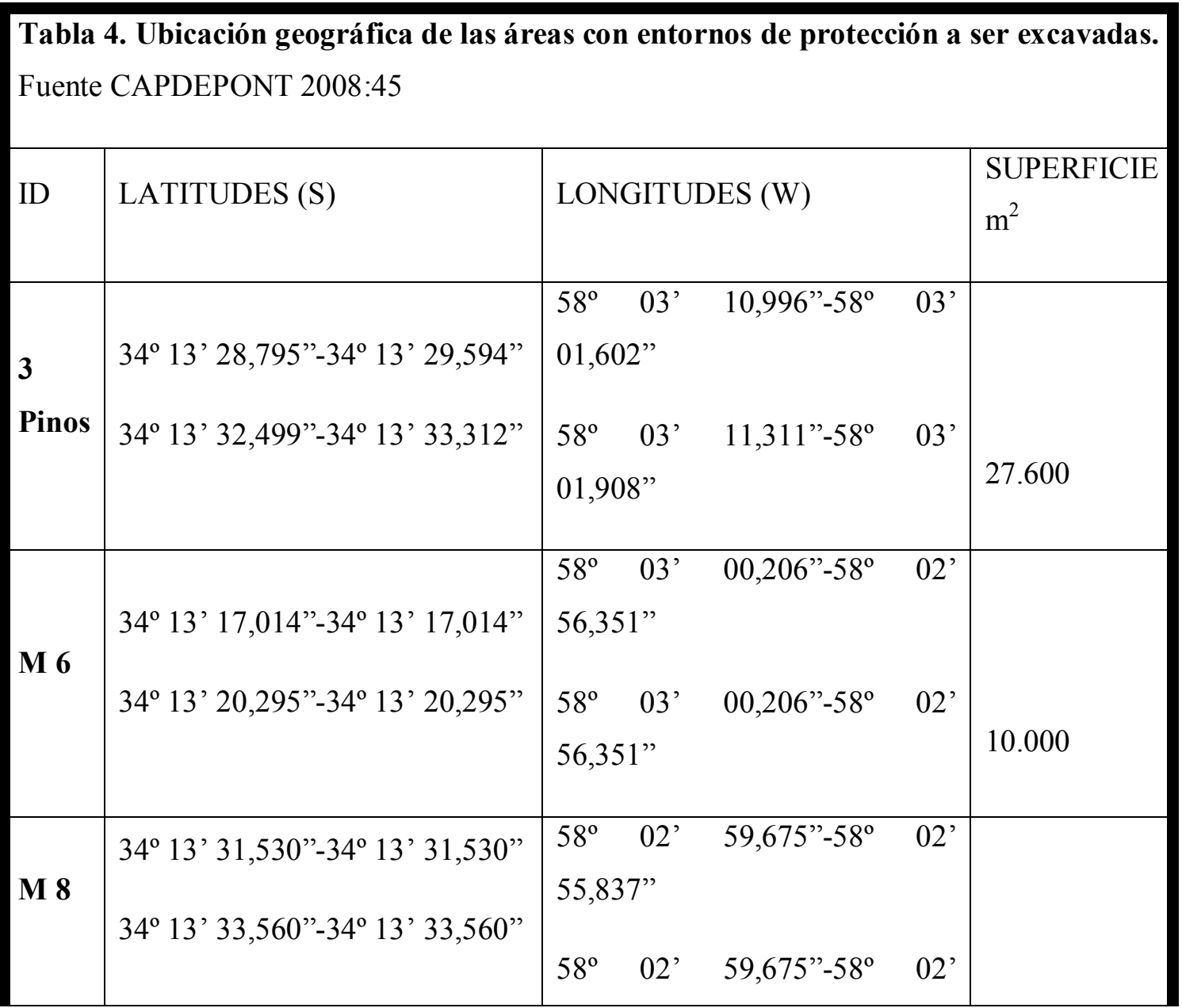


LAP Revista de Arqueologia Pública, n.8, Dezembro 2013. Campinas: LAP/NEPAM/UNICAMP.

\begin{tabular}{|c|c|c|c|c|c|}
\hline & & \multicolumn{3}{|l|}{$55,837^{\prime \prime}$} & 6.000 \\
\hline N 8 & $\begin{array}{l}34^{\circ} 13^{\prime} 34,765^{\prime} "-34^{\circ} 13^{\prime} 34,765^{\prime} \\
34^{\circ} 13 \text { ' } 35,431^{\prime \prime}-34^{\circ} 13^{\prime} 35,431^{\prime}\end{array}$ & $\begin{array}{l}58^{\circ} \quad 02^{\prime} \\
47,836^{\prime \prime} \\
58^{\circ} \quad 02^{\prime} \\
47,836^{\prime \prime}\end{array}$ & $\begin{array}{l}48,682^{\prime \prime}-58^{\circ} \\
48,682^{\prime \prime}-58^{\circ}\end{array}$ & $\begin{array}{l}02 ' \\
02\end{array}$ & 400 \\
\hline N 9 & $\begin{array}{l}34^{\mathrm{o}} 13^{\prime} 40,153^{\prime \prime}-34^{\mathrm{o}} 13^{\prime} 40,153^{\prime} \\
34^{\mathrm{o}} 13^{\prime} 40,689^{\prime}-34^{\mathrm{o}} 13^{\prime} 40,689^{\prime}\end{array}$ & $\begin{array}{l}58^{\circ} \quad 02^{\prime} \\
43,053^{\prime \prime} \\
58^{\circ} \quad 02^{\prime} \\
43,053^{\prime \prime}\end{array}$ & $\begin{array}{l}43,579^{\prime \prime}-58^{\circ} \\
43,579^{\prime \prime}-58^{\circ}\end{array}$ & $\begin{array}{l}02^{\prime} \\
02 '\end{array}$ & 225 \\
\hline
\end{tabular}

La superficie abarcada por el total de las excavaciones alcanzó los $286 \mathrm{~m}^{2}$. Paralelamente, se realizaron sondeos tendientes a caracterizar sectores de los sitios que no incluyeron excavaciones, cubriendo un total de $40 \mathrm{~m}^{2}$. La profundidad alcanzada en cada intervención y la cuantificación de materiales recuperados se observa en la tabla 5.

\begin{tabular}{|c|c|c|c|}
\hline \multicolumn{4}{|c|}{$\begin{array}{l}\text { Tabla 5. Datos generales de las intervenciones realizadas. Fuente: } \\
\text { CAPDEPONT 2008a,b; DEL PUERTO 2008a, b; GASCUE } 2008 .\end{array}$} \\
\hline Intervención & $\begin{array}{l}\text { Área } \\
\left(\mathrm{m}^{2}\right)\end{array}$ & $\begin{array}{l}\text { Profundidad } \\
\text { (cm) }\end{array}$ & $\begin{array}{l}\text { Total de Materiales } \\
\text { Líticos }\end{array}$ \\
\hline M6 Exc.I & 15 & 70 & 493 \\
\hline M6 S11-16 & 12 & $\sim 100$ & 15 \\
\hline 3P Exc.I & 25 & 180 & 36 \\
\hline 3P Exc.II & 20 & 150 & 1216 \\
\hline 3P Exc.III & 40 & 160 & 1571 \\
\hline 3P Exc.IV & 24 & 250 & 554 \\
\hline 3P Exc.V & 40 & 60 & 4566 \\
\hline
\end{tabular}




\begin{tabular}{|l|l|l|l|}
\hline 3P Exc VI & 6 & 460 & 431 \\
\hline 3P Exc VII & 4 & 110 & 1486 \\
\hline 3P S12- 19 & 24 & $\sim 80$ & 251 \\
\hline M8 ExcI & 35 & 240 & 9695 \\
\hline M8 Exc.II & 20 & 150 & 94 \\
\hline M8 Exc.III & 15 & 110 & 10684 \\
\hline M8 018 & 4 & 160 & 682 \\
\hline M8 S15- 17 & 12 & $\sim 80$ & 3 \\
\hline N8 Exc.I & 18 & 260 & 33 \\
\hline N9 Exc.I & 12 & 190 & 628 \\
\hline
\end{tabular}

A continuación se presentan los resultados obtenidos de las excavaciones realizadas en los espacios denominados como M8 y M6.

En M8 se plantearon las siguientes excavaciones: Exc I de $5 \times 7$ m, Exc. II de 5 x 4 m y Exc. III de 5 x $3 \mathrm{~m}$. En las mismas se observó que las unidades estratigráficas superiores (UE01, UE02 Y UE03) presentaban materiales culturales en contextos secundarios. Ello es producto de la remoción ocasionada por actividades de extracción de áridos en el pasado histórico del Punta Pereira. A partir de la UE05, en las excavaciones realizadas comenzó a recuperarse material lítico en contexto primario. En las UE06 y UE07 se observó la presencia de materiales líticos, estructuras sedimentarias (manchas) y estructuras de combustión (fogones). Este tipo de registro se encontró particularmente concentrado en la UE07. El evento de ocupación humana evidenciado en la UE07, aportó una cronología de $4.240 \pm 80$ años ${ }^{14} \mathrm{C}$ AP (URU0505) (BRACCO 2008) obtenida a partir de material vegetal carbonizado de una de las estructuras de combustión. En la unidad estratigráfica UE07 se recuperó el 81\% de los materiales arqueológicos $(n=21.158)$ asociados a fogones (Figura 5). El material cultural evidencia una tecnología lítica de reducción y fractura expeditiva. La misma se habría basado en la obtención de formas base, para ser seleccionadas como instrumentos (MAROZZI et al., 2008). En los materiales se encuentran representadas actividades de manufactura, uso y descarte, observándose en el conjunto una baja diversidad de artefactos y 
la presencia de marcas de uso en algunos de los mismos. Las materias primas utilizadas en la manufactura de estos materiales, como cuarzo y granito, se encuentran accesibles en fuentes primarias y secundarias del entorno inmediato. También se ha observado el uso de materias primas regionales como la caliza silicificada (MAROZZI et al., 2008; CAPDEPONT, 2012).

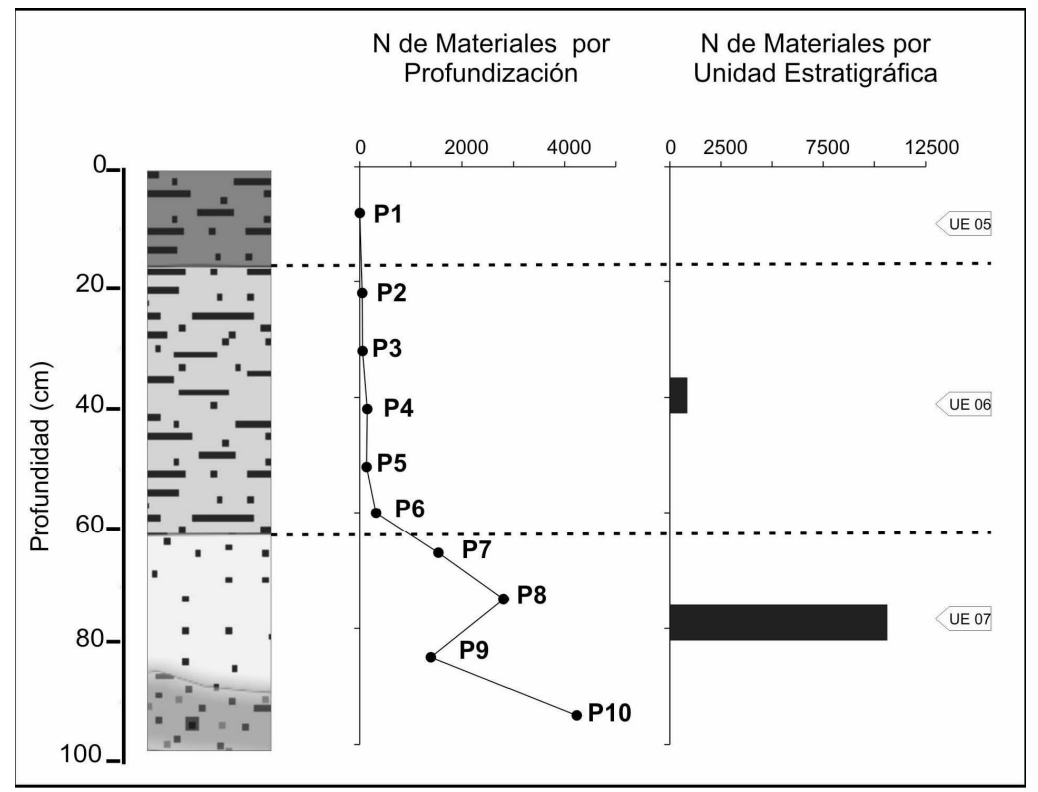

Fig.5: Representación del perfil tipo de M8 con total de materiales líticos recuperados. Fuente: DEL PUERTO 2008a: 314.

De acuerdo a los resultados obtenidos, en la unidad del paisaje correspondiente a un antiguo arco de playa, se desarrollaron intensas actividades de ocupación humana hacia el Holoceno medio-tardío. Se cuenta con evidencias que llevan a considerar que en este espacio se realizaron actividades relacionadas a la manufactura y/o mantenimiento de instrumentos líticos en directa asociación con fogones. Dados los datos manejados, también puede proponerse que los restos recuperados fueron generados en un campamento base o residencial (sensu BINFORD, 1980), ubicado próximo a la línea de costa de hace 4000 años.

Las intervenciones realizadas permitieron una amplia caracterización geoarqueológica de este sector, relevando las secuencias estratigráficas e identificando las áreas de concentración de vestigios arqueológicos. En este sentido, se destaca el carácter del paleosuelo arenoso como estrato guía, pero disociado en su expresión vertical de la concentración de materiales culturales. Los sectores donde el paleosuelo se encuentra presente y mejor definido son los que cuentan con mayor concentración de materiales, a pesar de que los mismos se hallan mayormente comprendidos en unidades estratigráficas subyacentes. 
En las intervenciones realizadas en M6 se recuperaron materiales líticos en un paleosuelo limo-arcilloso de origen aluvial cubierto por depósitos eólicos modernos. El mismo se encuentra ubicado en una hollada interdunar natural cubierta por vegetación arbórea nativa. En las excavaciones realizadas se registraron seis unidades estratigráficas que se corresponden a cinco horizontes de suelo: A0 (UE01), A1 (UE02-03), A2 (UE04), B1 (UE05) Y B2 (UE06). Las dos primeras unidades estratigráficas resultaron arqueológicamente estériles, a excepción de una pequeña lasca en cuarzo. En forma similar, de la UE 03 se recuperó una única lasca en caliza silicificada. En contraste, las tres unidades estratigráficas inferiores presentaron mayores concentraciones de material arqueológico. Fueron recuperadas 492 piezas líticas y un único fragmento óseo. Como ilustra la figura 6, los materiales se concentraron por debajo de los depósitos eólicos más recientes (UE01 A 03), en lo que conforma la matriz de un paleosuelo arcillo-limoso de origen aluvial. Las mayores concentraciones de materiales se recuperan de la UE05. Si bien no se cuenta con dataciones radiométricas que contextualicen temporalmente el registro arqueológico abordado, sus características geoarqueológicas (unidad de paisaje en la que se emplaza, cota, tipo de suelo) y tecnológicas (predominio de caliza silicificada como materia prima), sugieren que es el producto de una ocupación humana temporal y espacialmente diferente a las manifestadas en los restantes contextos arqueológicos intervenidos en Punta Pereira (DEL PUERTO, 2008b).

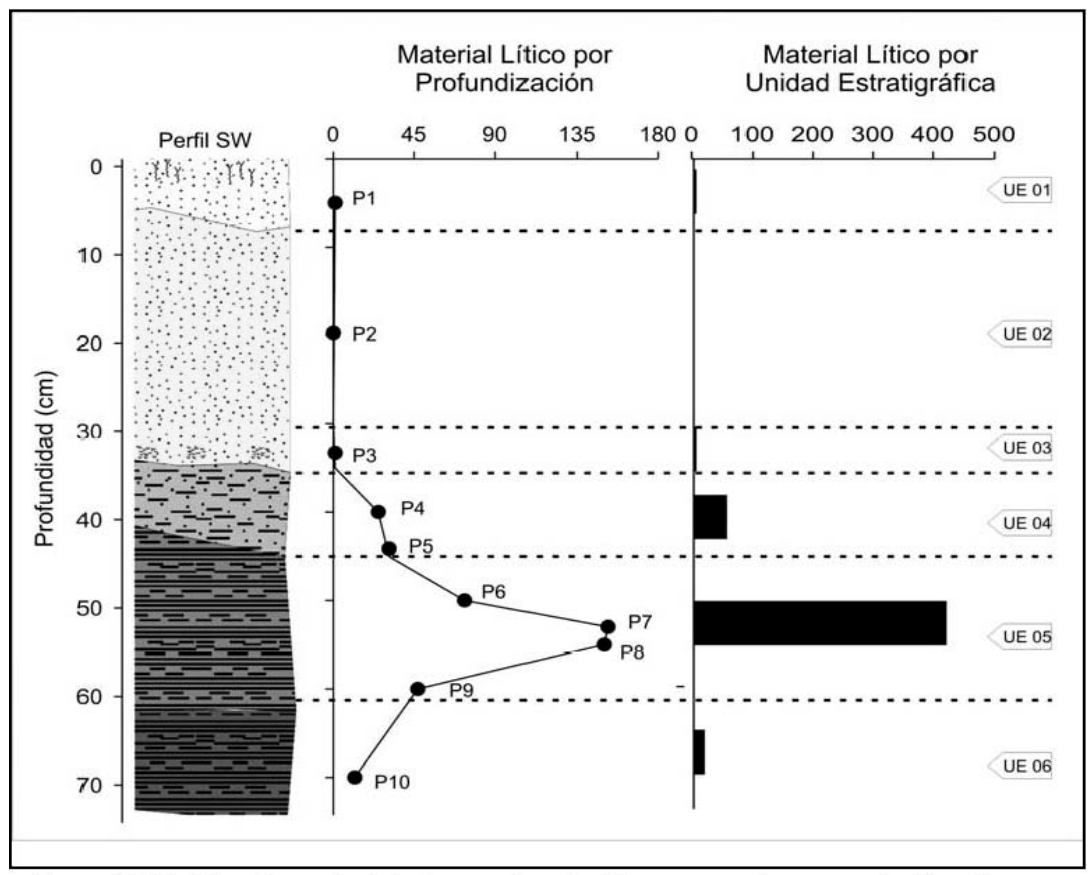

Fig.6: Distribución vertical de materiales líticos de M6 por profundización y UE. Fuente DEL PUERTO 2008b:26. 


\section{Consideraciones finales}

En primer lugar, es pertinente destacar que la implementación de medidas de corrección compensatorias (documentación, prospección y excavación) permitió mitigar el impacto a generarse en Punta Pereira. Ante la imposibilidad de evitar dicho impacto, dada la fase avanzada del trámite de habilitación ambiental en la que se requirió el estudio de impacto arqueológico, se desarrolló una estrategia tendiente a compensar sus efectos con la generación de información calificada que permitiera un mayor conocimiento del pasado prehistórico e histórico en la región. En este sentido, el cúmulo de datos generados evidencia que en Punta Pereira tuvieron lugar ocupaciones humanas prehistóricas en distintas unidades del paisaje. Una de ellas, con mayor expresión de cultura material en contexto primario al momento del estudio, se vincula espacialmente a la línea de la paleocosta del máximo transgresivo del Holoceno. Vinculada a la anterior, en cotas inferiores habría tenido lugar una ocupación más tardía, cuyos contextos han sufrido el mayor impacto de la antigua explotación minera. En tercer lugar, se obtuvieron evidencias de contextos primarios en cotas más elevadas, que podrían corresponder a asentamientos más tempranos.

Dentro de los sitios abordados en la línea de la paleocosta, la secuencia estratigráfica arqueológica puso en evidencia la existencia de al menos dos momentos de ocupación prehistórica. El primero tuvo lugar hacia el 4200 años ${ }^{14} \mathrm{C}$ AP., cuando el nivel del mar se encontraba por encima de su nivel actual, próximo al emplazamiento de los sitios. Las ocupaciones humanas habrían tenido lugar en dos unidades de paisaje principales. Por un lado, el sitio 3Pinos se ubica en lo que fue una península rocosa, mientras que los restantes sitios investigados se localizan en lo que fue un arco de playa al este de la península. La cultura material recuperada, los rasgos y estructuras registrados, indicarían ocupaciones vinculadas a campamentos base. Ello también se sustenta con la información regional, obtenida de diversas investigaciones realizadas en la costa atlántica, que manifiestan una secuencia estratigráfica en extremo similar (LÓPEZ, 1994, 1995; LÓPEZ et al, 2003-2004).

Depósitos similares a los de la escarpa de Punta Pereira, también subyacen a un paleosuelo en sitios de Cabo Polonio (LÓPEZ, 1994), sometidos a una batería de fechados que los coloca entre 4500 y 4100 años ${ }^{14} \mathrm{C}$ AP. Estos potentes depósitos (de aproximadamente $1 \mathrm{~m}$ ) vinculados a una fase árida, parecen haberse depositado en la región durante un período acotado. En este sentido, existe un patrón de ocupación humana similar en toda la costa oceánica y estuárica de Uruguay. Este patrón se vincula a estrategias de subsistencia de explotación de recursos costeros bajo condiciones ambientales más áridas y con un nivel del 
mar por encima del nivel actual. Lo mencionado anteriormente permite proponer la presencia del paelosuelo como estrato guía para ubicar y contextualizar las ocupaciones del Holoceno medio-tardío.

En estos mismos sitios, en unidades estratigráficas superiores relacionadas al desarrollo del mencionado paleosuelo areno-limoso, se registraron evidencias de una ocupación más tardía con una edad mínima de 265 años ${ }^{14} \mathrm{C}$ AP (BRACCO, 2008). Es de destacar que un paleosuelo areno limoso fue observado en numerosos puntos de la costa uruguaya y en la misma cota, lo que permite considerarlo también como estrato guía. Este fue fechado en Cabo Polonio (sobre carbón cultural) entre ca 700 y 600 años ${ }^{14} \mathrm{C}$ AP. (LÓPEZ, 1994), lo cual permite afinar la fecha obtenida para esta unidad estratigráfica. La menor concentración de cultura material recuperada en esta unidad evidenciaría una ocupación más efímera o relictual en el paisaje. En este sentido, la presencia de paleosuelo removilizado con material cultural en pilas estériles generadas por la antigua actividad minera, indica que el paleosuelo se desarrollaba hacia la costa, finalizando su desarrollo horizontal a pocos metros al norte de la escarpa. Esta ocupación más tardía habría tenido lugar con un nivel del mar similar al actual y podría vincularse a los hallazgos cerámicos recuperados en superficie en la zona baja. Las intervenciones efectuadas en el sector M6 denotaron la existencia de contextos arqueológicos posiblemente más antiguos. La ubicación de estos contextos en cotas más altas (15 msnm), en unidades estratigráficas diferentes y con una tecnología lítica caracterizada por el predominio del uso de materias primas como la caliza silicificada, respondería a una ocupación humana más temprana.

La implementación del Estudio de Impacto Arqueológico en Punta Pereira generó información que enriquece el conocimiento de la prehistoria local y regional, demostrando que es posible compatibilizar el avance productivo con la protección y puesta en valor del patrimonio cultural prehistórico.

\section{Referencias bibliográficas}

AMADO Xuxo, BARREIRO David, CRIADO Felipe y MARTÍNEZ María. Especificaciones para una gestión del Impacto desde la Arqueología del Paisaje. TAPA 26, 2002.

BRACCO, Roberto. Informe del laboratorio - Datación ${ }^{14} \mathrm{C}$. In: Informe Final del Estudio del Impacto Arqueológico y Cultural de la Construcción de la Fabrica de Celulosa y Planta de Energía Eléctrica de Punta Pereira. Tomo III La Prehistoria, Capítulo 21, pp. 369-374. Facultad de Humanidades y Ciencias de la Educación. UR. 2008

BINFORD, Lewis. "Willow Smoke and Dogs Tails: Hunter-gatherer Settlement Systems and 
Archaeological Site Formation" In: American Antiquity 45(1): 4-20. 1980

CAPDEPONT, Irina. Arqueologia de sociedades indígenas del litoral del Rio Uruguay. 2012. Tesis Doctoral em Arqueología. Facultad de Ciencias Sociales - Universidad del Centro de la Provincia de Buenos Aires, Olavarria. 2012

CAPDEPONT, Irina. Introducción. In: Informe Final del Estudio del Impacto Arqueologico y Cultural de la Construcción de la Fabrica de Celulora y Planta de Energía Eléctrica de Punta Pereira. Tomo I, Capítulo 1. Intervenciones en Arqueología Prehistórica, pp. 35-54. Facultad de Humanidades y Ciencias de la Educación. UR. 2008

CAPDEPONT, Irina. M8 Excavación I. In: Informe Final del Estudio del Impacto Arqueologico y Cultural de la Construcción de la Fabrica de Celulora y Planta de Energía Eléctrica de Punta Pereira. Tomo III La Prehistoria, Capítulo 15, pp. 231-262. Facultad de Humanidades y Ciencias de la Educación. UR. 2008a

CAPDEPONT, Irina. N8 excavación I. In: Informe Final del Estudio del Impacto Arqueologico y Cultural de la Construcción de la Fabrica de Celulora y Planta de Energía Eléctrica de Punta Pereira. Tomo III La Prehistoria, Capítulo 19, pp. 321-343. Facultad de Humanidades y Ciencias de la Educación. UR. 2008b

CRIADO Felipe, VILLOCH Victoria y BARREIRO David. Arqueología y Parques Eólicos en Galicia: Proyecto Marco de Evaluación de Impacto. CAPA n ${ }^{\circ}$ 5. 2000

DEL PUERTO, Laura. M8 Excavación III. In: Informe Final del Estudio del Impacto Arqueologico y Cultural de la Construcción de la Fabrica de Celulora y Planta de Energía Eléctrica de Punta Pereira. Tomo III La Prehistoria, Capítulo 18, pp. 291-317. Facultad de Humanidades y Ciencias de la Educación. UR. 2008a

DEL PUERTO, Laura. M6 Excavación I. In: Informe Final del Estudio del Impacto Arqueologico y Cultural de la Construcción de la Fabrica de Celulora y Planta de Energía Eléctrica de Punta Pereira. Tomo III La Prehistoria, Capítulo 1, pp. 3-32. Facultad de Humanidades y Ciencias de la Educación. UR. 2008b

GASCUE, Andrés. "Intervenciones en el sitio Tres Pintos" In: Informe Final del Estudio del Impacto Arqueológico y Cultural de la Construcción de la Fabrica de Celulosa y Planta de Energía Eléctrica de Punta Pereira. Tomo III La Prehistoria, Capítulo 2, pp. 34-45. Facultad de Humanidades y Ciencias de la Educación. UR. 2008.

LEZAMA, Antonio; DEL PUERTO, Laura; CAPDEPONT, Irina; INDA, Hugo; GASCUE, Andres; LEMBO, Victoria y CAMORS, Veronica. Plan de Actuación - Estudio de Impacto Arqueológico y Cultural en Punta Pereira. Facultad de Humanidades y Ciencias de la Educación. Expedientes: DINAMA 2007/14000/05626; CNPCN No 2007/ 678, 86pp. 2007

LEZAMA, Antonio; CAPDEPONT, Irina; DEL PUERTO, Laura; GASCUE, Andres; INDA, Hugo; BAEZA, Jorge; VIENNI, Bianca; BRUM, Laura; LÓPEZ, Enrique; CAMORS, Veronica; ROMERO, Sonia; LEMBO, Victoria y PINTOS, Mariana. Diagnostico del estudio de Impacto Arqueológico y Cultural del área Terrestre de la Construcción de la Fábrica de Celulosa y Planta de Energía Eléctrica de Punta Pereira. Facultad de Humanidades y 
Ciencias de la Educación, UR. 2008.

LÓPEZ, José María. "Cabo Polonio: Sitio Arqueológico del Litoral Atlántico Uruguayo" In: Revista de Arqueología, Anais da VII Reuniao Científica da Sociedade de Arqueología Brasileira, 8 (2): 333-353, San Pablo. 1994

LÓPEZ, José María. "El Fósil que no Guía, y la Formación de los Sitios Costeros" In: Arqueología en el Uruguay, editado por M. Consens, J.M. López Mazz y C. Curbelo, pp. 92105. Surcos srl, 1995

LÓPEZ, José María; GASCUE, Andrés y MORENO, Federica. "La Prehistoria del Este de Uruguay: Cambio Cultural y Aspectos Ambientales" In: Anales de Prehistoria y Arqueología, 19-20: 9-24. 2003-2004.

MAROZZI, O; SOTELO, Moira; GAZZAN, Nicolás; FERRARI, Alejandro y Gastón Lamas. "Análisis del material lítico" In: Informe Final del Estudio del Impacto Arqueológico y Cultural de la Construcción de la Fabrica de Celulosa y Planta de Energía Eléctrica de Punta Pereira. Tomo III La Prehistoria, Capítulo 23, pp. 403-478. Facultad de Humanidades y Ciencias de la Educación. UR. 2008. 